\title{
Kooperation zwischen Umwelt- und Entwicklungsverbänden
}

\author{
Eine Zusammenarbeit zwischen Umwelt- und \\ Entwicklungsverbänden besteht in Deutsch- \\ land spätestens seit der 1992er-Konferenz der \\ Vereinten Nationen über Umwelt und Entwick- \\ lung. Maßgeblich wird diese im Netzwerk Forum \\ Umwelt\& Entwicklung koordiniert. Welche \\ Erfolgsbedingungen gibt es für diese Zusammen- \\ arbeit? Von Marie-Luise Abshagen
}

D as Forum Umwelt Q Entwicklung, ein Netzwerk deutscher Nichtregierungsorganisationen (NGOs), koordiniert deren Zusammenarbeit in internationalen Prozessen zum Thema Umwelt und Entwicklung. Seine Gründung fällt in den Zeitraum der 1992er-Konferenz der Vereinten Nationen (UN) über Umwelt und Entwicklung (Rio-Konferenz), bei der erstmals im Rahmen internationaler Verhandlungen über die Notwendigkeit des Zusammendenkens beider Bereiche diskutiert wurde.

\section{Besondere Mitgliedsstruktur des Forums}

Seit Gründung 1992 arbeiten Nichtregierungsorganisationen (NGOs) aus den beiden Politikfeldern Umwelt und Entwicklung im Forum Umwelt \& Entwicklung. Die Zusammenarbeit erfolgt entweder als offizielles Mitglied oder als lose assoziierter Verband. Diese Doppelstruktur ermöglicht es dem Forum, eine Vielzahl unterschiedlich großer Verbände zusammenzuhalten. Verbände erhalten durch die Mitgliedschaft das Recht, den umwelt- und entwicklungspolitisch paritätisch besetzten Leitungskreis zu wählen oder darin vertreten zu sein. Dafür wird je nach Finanzstärke ein Mitgliedsbeitrag fällig. Verbände, die beispielsweise aufgrund ihres eigenen Mandats nicht Mitglied in anderen Verbänden sein können, unterliegen nicht diesen Rechten und Pflichten, eine Mitarbeit im Forum ist aber ebenfalls möglich. Hierin unterscheidet sich das Forum als aus politischem Bedarf gewachsenes Netzwerk von formellen Dachverbänden, da es eine niedrigschwelligere Mitarbeit ermöglicht.

Die Zusammenarbeit von Verbänden wird durch Arbeitsgruppen (AGs) ermöglicht. In den zahlreichen, thematischen AGs begleitet das Forum internationale Prozesse, die sich mit Umwelt- und Entwicklung befassen. Jede AG hat einen oder zwei Sprecher/innen, die aus den in der AG aktiven Verbänden stammen und von der AG benannt werden. Zudem wird jede AG von einer Mitarbeitenden aus der Geschäftsstelle unterstützt.
Die zu bearbeitenden Themen setzen die Verbände selber. Während einige AGs schon seit Gründung des Forums existieren, unterliegt die Zusammenarbeit auch einer gewissen Dynamik. Ergeben sich neue Themen, können Verbände entscheiden, neue AGs zu gründen. Hat ein Thema politisch keine Relevanz mehr, werden AGs auch nicht weiter betrieben. Die Entscheidungen für Neugründung und Auflösung fällen je nach Gemengelage die Verbände in den AGs, die Geschäftsstelle oder der Leitungskreis.

Darüber hinaus werden Themen von der Geschäftsstelle an die Verbände und die AGs herangetragen. Welche Themen die Geschäftsstelle bearbeitet, entscheidet in letzter Konsequenz der Leitungskreis. Dieses Gremium tagt vierteljährlich und bespricht abgeschlossene und künftige Arbeitsprozesse.

\section{Dynamische Formen der Zusammenarbeit}

Anlassbezogen bilden sich auch außerhalb von AGs dynamischere Strukturen der Zusammenarbeit. Dies ist insbesondere bei jenen Themen der Fall, die besonders vielseitig sind. Dazu gehört beispielhaft die Verhandlung der Sustainable Development Goals (SDGs). Den Nachhaltigkeitszielen gingen im September 2015 ein Beschluss der Rio+20-Konferenz und drei darauffolgende Verhandlungsjahre voraus. Als Prozess der Vereinten Nationen (UN) primär von Staatsvertreter/innen angetrieben, zeigte sich dieser Verhandlungsprozess als ungewöhnlich offen für zivilgesellschaftliche Stimmen. Aufgrund dieses Austausches zwischen UN-, Staaten- und Zivilgesellschaftsvertretern bildete sich spätestens ab 2013 weltweit viel Engagement und Mitgestaltungswille bei NGOs heraus. Kurz nach der Rio+20Konferenz zeigte auch die deutsche Zivilgesellschaft hohes Interesse, die Verhandlung der SDGs inhaltlich zu begleiten. Die SDG-Verhandlungen stellten eine besondere Chance dar, jenseits bestehender Verbändepositionen sektor- und themenübergreifende Schwerpunkte in einen internationalen Text zu setzen.

Dem Forum Umwelt \& Entwicklung kam aufgrund seiner langen Historie in der Koordinierung entwicklungs- und umweltpolitischer Belange eine besondere Rolle zu. In der Geschäftsstelle des Forums wurde eine halbe Stelle geschaffen, die sich ausschließlich mit den UN-Verhandlungen und der Erarbeitung einer Verbändepositionierung befasste. Da viele mit den Millenium Development Goals (MDGs) vertraute Organisationen bereits an entwicklungspolitischen Positionen arbeiteten, fiel innerhalb der Forumsstrukturen die Entscheidung, umweltpolitische Ziele für die neue Agenda zu formulieren, die aber 
auch entwicklungspolitische Ziele berücksichtigten. Unter intensiver Einbindung von Verbänden wurde das im November 2013 veröffentlichte Positionspapier Die ökologische Dimension in der Post-2015-Agenda für nachhaltige Entwicklung erstellt.

\section{Grundbedingungen erfolgreicher Zusammenarbeit}

Zusammenarbeit zwischen Verbänden kommt vor allem anlassbezogen zustande. Dieser Anlass wird idealerweise durch einen institutionellen Prozess oder eine politische Dringlichkeit bedingt, wodurch eine Notwendigkeit bei Verbänden entsteht, entsprechende Positionierungen auszuarbeiten.

Der Anlass kann dabei entweder in einem bereits bestehenden Verfahren verankert sein, beispielsweise eine neue Verhandlungsrunde in einem bestehenden UN-Prozess. Gleichermaßen ist das Auftauchen eines relativ neuen Themas oft eine Möglichkeit für Verbände, überhaupt erst eine Positionierung zu erarbeiten oder alte Positionen abzugleichen und gegebenenfalls anzupassen.

\section{Mandat und Maximierung von Lobbyerfolgen}

Existieren Möglichkeiten der Einflussnahme, entsteht der Bedarf innerhalb der NGOs zu einem bestimmten Thema $\mathrm{zu}$ arbeiten. Dies ist natürlich nicht der einzige Faktor, auch die Dringlichkeit eines Themas ebenso wie die ureigene Aufgabe des Verbandes, zu bestimmten Themen zu arbeiten, sind Grund für neues oder aufgewertetes Handeln. Nichtsdestotrotz bleibt die Möglichkeit, auf politische Prozesse einzuwirken, wichtige Treibkraft für NGOs, deren Aufgabe u. a. Lobbyarbeit umfasst.

Insbesondere bei Themen, denen eine Überschreitung des eigenen Mandats und/oder eine deutliche Überschneidung verschiedener Verbandsmandate inneliegt, sehen Verbände oft einen Mehrwert für Kooperation. Begründet wird dies verschiedentlich, sei es durch Fachexpertisenaustausch, Erfolgsmaximierung in der Lobbyarbeit oder grundsätzliche Offenheit für Zusammenarbeit als effektiveres Arbeitstool.

Hinzu kommt der Umgang mit Zielkonflikten (z. B. Schutz vs. Nutzung) innerhalb der Verbandsszene. Deren Thematisierung oder Auflösung liegt zumeist begründet in politisch-strategischen Verbandsüberlegungen oder aber auch neuen wissenschaftlichen Erkenntnissen. Daraus ergibt sich der Wille zur Kooperation, um Zielkonflikte zu diskutieren und gegebenenfalls in gemeinsamen Positionen aufzuheben.

\section{Förderung von Kooperation}

Kooperation mit Akteuren außerhalb der eigenen Organisation oder Szene wird auch von außen begünstigt. Das meint sowohl prozedurale Strukturen der Zusammenarbeit (z. B. Internationale Organisationen stellen Themenforen bereit) oder durch vom politischen Gegenüber gesetzte Bedingungen (z. B. Regierungen fordern abgestimmte NGO-Position). Sowohl das
Agieren innerhalb dieser vorgegebenen Bedingungen wie auch dessen Ablehnung kann zu Kooperation führen.

\section{Klare Rollen und Unterstützung durch bestehende Strukturen}

Eine vorhandene Verbändekoordinationsstruktur wird dann genutzt, wenn von beteiligten Verbänden darin ein Mehrwert für die Zusammenarbeit erkannt wird. Darunter fallen Strukturen, die inhaltlichen Austausch, Absprachen und gemeinsame Öffentlichkeits- und Lobbyarbeit vereinfachen, begleiten und gegebenenfalls finanzieren. Ein zentraler Erfolgsfaktor ist eine feste Struktur (wie bspw. die Geschäftsstelle des Forums), die Verbänden Koordinationsarbeit abnimmt durch Unterstützung bei allen aus Koordinationsstrukturen entstehenden Arbeitsaufträgen (z. B. Organisation von Sitzungen, gemeinsamen Veranstaltungen oder Publikationen).

Bestehende institutionelle Grundlagen, innerhalb derer Zusammenarbeit stattfindet beziehungsweise die Zusammenarbeit unterstützen, können Kooperation bedingen und fördern. Einfluss hat auch eine durch das Mandat der Kooperationsstruktur definierte Mitgliederstruktur. So ist zum Beispiel durch die ureigene Aufgabe des Forums, Umwelt- und Entwicklungsverbände zusammenzubringen, eine Zusammenarbeit zwischen diesen Gruppen gesetzt. Feste Entscheidungsstrukturen für die Verbände (beim Forum der Leitungskreis und die AGs) garantieren angemessene Einbindung der Mitglieder. Erfahrung mit Mitgliedern kann der Kooperationsstruktur zudem besondere Fähigkeiten und Rollen in der Moderation verschiedener Verbandspositionen geben, was potenzielle Konflikte abschwächt. Die Art der Kooperationsstruktur kann ein maßgeblicher Faktor in der Einbindung von Akteuren sein. Während Dachverbände tendenziell formalisiertere Strukturen haben und beispielsweise Anträge zur Mitarbeit oder Veränderung der Strukturen gestellt werden müssen, kann die Form eines Netzwerkes, wie im Falle des Forums, flexibler für Zusammenarbeit sein. Mitgliedschaft und Entscheidungsstrukturen sind in diesem Fall offener. Nachteile darin könnten Hindernisse bei Antragsstellungen sein, die zum Beispiel Vereinsstrukturen fordern oder Anerkennung durch politische Akteure. Beides ist jedoch bei Forum nicht der Fall.

Schlussendlich liegt ein nicht zu unterschätzender Faktor in der Verbandskooperation darin, dass deren Mitarbeitende sich häufig durch ein hohes $\mathrm{Maß}$ an persönlichem Engagement auszeichnen, dem oft das Interesse an Zusammenarbeit mit anderen inhärent ist.

AUTORIN + KONTAKT

Marie-Luise Abshagen ist Referentin für nachhaltige Entwicklung beim Forum Umwelt \& Entwicklung.

Forum Umwelt und Entwicklung, Marienstr. 19-20 10117 Berlin. Tel: +4930678177575. E-Mail: abshagen@forumue.de Website: www.forumue.de

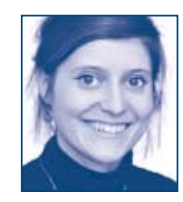

